\title{
Review on Gray Track Effects on Potassium Titanyl Phosphate Single Crystals
}

\author{
Narayana Perumal R*, Nallathambi A and Subramani S \\ Center for Radiation Environmental Science and Technology, SSN College of Engineering, India
}

Submitted: November 29, 2017; Published: December 05, 2017

*Corresponding author: Narayana Perumal R, Center for Radiation Environmental Science and Technology, SSN College of Engineering, Kalavakkam-603110, India, Email: rajeshnp@ssn.edu.in; rajeshnp@hotmail.com

\begin{abstract}
Potassium titanyl phosphate (KTP) is an interesting crystal for many applications in nonlinear optics and electro-optics. Its large optical nonlinearity, damage resistance, wide acceptance angle, low insertion loss and thermally phase-matching properties make KTP crystal suitable for frequency doubling of infrared lasers. In addition, it is chemically inert and mechanically robust which are important in the construction of reliable devices suitable for industrial and medical applications. Though this crystal has enormous functional advantage, the charge defect known as gray tracking reduces its efficiency. This article aims to provide detail concept of gray tracking that mainly involves mechanism that lead to initiation of gray track, by laser and electric field. Discussed the dependency factors like wavelength, threshold, moisture, and repetition rate of laser, dopant addition, polarisation that influence the gray track to signify the effects of gray tracking in KTP and discoursed the measures to control and eliminate the gray tracking.
\end{abstract}

Keyword: Second Harmonic Generation (SHG)

\section{Introduction}

The contemporary trend in industrial and medical fields is to adopt solid state lasers with high average power, this requires technologies of higher harmonics generation with efficiency and stability, the efficiency of nonlinear crystal long-wavelength laser emissions to the visible and ultraviolet spectral ranges has led to a constant increasing use of these crystals. Among them KTP is one of the nonlinear crystal frequently opted for its excellent properties like a large nonlinear optical coefficient, wide acceptance angles, thermally stable phase-matching additionally to its attractive nonlinear optical characteristics, It is also utilized in electro-optic applications such as waveguide modulators and Puckers cells because of its large electro-optic coefficients and low dielectric constants, These high efficiency and stability is sufficient to fulfil the expectancy. However this crystal suffers from an important disadvantage, that is the formation of the colour centre called gray tracking.

Gray tracking is a fatigue damage arises due to defect in crystal characterised by a coloured appearance in them which is commonly known as darkening that reduces the non-linear property of crystal through optical power losses(by absorption) that is said to be as chromic damage and so as lowering its performance in applications. Mostly flaws in crystal will decrease the efficiency of their property, here quoted some illustration to showcase effect in crystal due to this damage, Beno^lt Boulanger
[1], experiments show that gray tracking modifies the parametric gain and also many experiments proved that this damage lowers the SHG efficiency and causes astigmatism of the output laser beam and limits the overall lifetime of optical elements, So as if gray tracks once initiated, absorb a significant portion of the propagating beams causing optical power losses and continued operation often leads to catastrophic damage especially in KTP $\left(\mathrm{KTiOPO}_{4}\right)_{[2,3]}$. The scope of this paper is concerned with propagating the overall knowledge of gray tracking including their mechanism and effects.

To implicate the idea in simple terms let's say that Ionizing radiation such as laser beam or x-rays is capable to produces electrons and holes that become separately trapped within the lattice of KTP thus forms colour centres. Thus classification is done based on two sources nothing but both the laser and electric-field which is its prime application, by inducing damage susceptibilities. Depend on the source they are named as photo chromic damage and electro chromic damage. This colouration phenomenon generally observed in KTP crystals during the second harmonic generation (SHG). Some possible mechanisms that lead to the formation of these electron-hole pairs during SHG are effects of sum-frequency generation, one-photon absorption and multi-photon absorption. However, the damage susceptibilities of the crystals also depend on the growth technique. Many different hypotheses have been presented 
to explain this phenomenon, which will be seen in following sessions.

\section{Mechanism Involved in Gray Tracking Formation}

Focus of many investigators is to recover the KTP from gray tracking to produce assured product for industrial use, To do so the core cause of gray track should be known, though various susceptibility is feasible, general believe is that defects that trap photo induced electron and holes is the primary engender, the defect mentioned is trapping sites, Optical absorption and electron paramagnetic resonance [EPR] studies can be opted to identify the trapping sites. Few investigators worked on in it namely Roelofs [4] described four distinct $\mathrm{Ti}^{3+}$ through EPR Spectral study in electrically induced or annealing in a hydrogen atmosphere in flux grown crystal, flowingly Andreev and Asimov found the same effect in X-ray irradiation at room temperature also Andreev [5] demonstrated the same using Laser beam. Likely the application themselves efficient to produce trapping sites. Elimination of these trapping sites considerably increases the gray track damage threshold. One can easily understand the mechanism with the description of X.B. Hua [6] as of the laser irradiated in KTP, some electron-hole pairs will be produced in the perfect lattice.

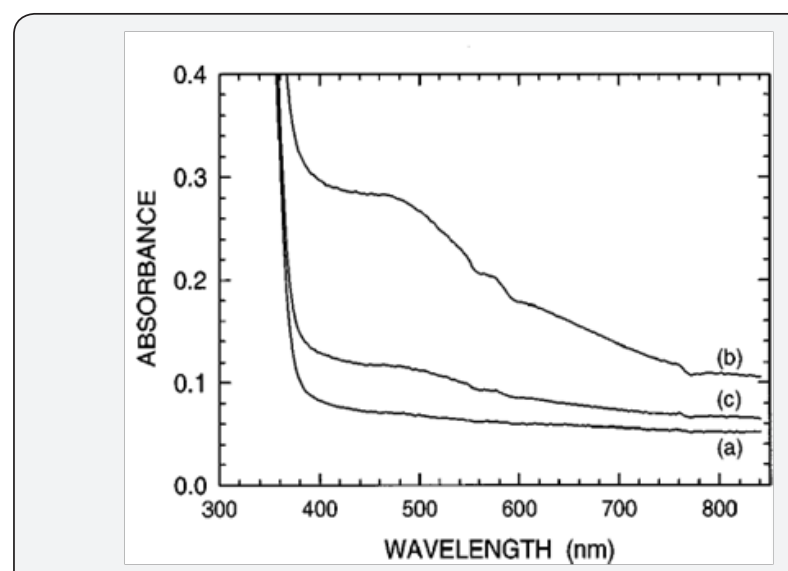

Figure 1: Optical absorption spectra of flux grown KTP taken at room temperature.

(a) Before irradiation,

(b)After irradiation at0 oc,

(c) $9.5 \mathrm{~h}$ after the radiation [8].

Many of the electrons and holes will immediately recombine to restore the perfect lattice; but, a portion of the electrons and holes will migrate sufficiently far from each other and encounter a stabilizing entity, either a vacancy or an extrinsic impurity. Thus, a few of the electrons and holes will be stabilized at widely separated sites within the crystal and form the point defects (optical absorption centres) that contribute to the formation of a gray track [6]. Primal scholars $[1,4,7]$ suggested that $\mathrm{Ti}^{3+}$ may cause optical damage but this didn't give any direct relation to the cause of gray tracking, there after Scripsick MP et al.[8] have overcome above statement, by experimentally proving that $\mathrm{Fe}^{3+}$ traps holes and $\mathrm{Ti}^{3+}$ traps electron, Nizamutdinov et al.
[9]. Experiments proved existence of EPR spectra $\mathrm{Fe}^{3+}$ in KTP for the first time following him Stenger et al. [10] and Gaite [11] distinguished four spectra of $\mathrm{Fe}^{3+}$ in flux grown KTP, latter studies suggested that $\mathrm{Fe}^{3+}$ occupy $\mathrm{Ti}(1)$ and $\mathrm{Ti}(2)$ sites depend on crystal and ENDOR(electron-nuclear double resonance) studies by Scrip sick suggested that oxygen vacancies are located adjacent to the $\mathrm{Ti}^{3+}$ centres also (Figures 1 \& 2) [8] depict EPR results conformance with all these theory basis they forwarded to trapping sites identification in flux grown crystal and induced gray track through $\mathrm{x}$-ray source where the effect was found at 500nm due to $\mathrm{Fe}^{3+}$ other negligible effect due to $\mathrm{Fe}^{2+}$ or $\mathrm{Fe}^{4+}$ also seen in (Figure 1) [8].

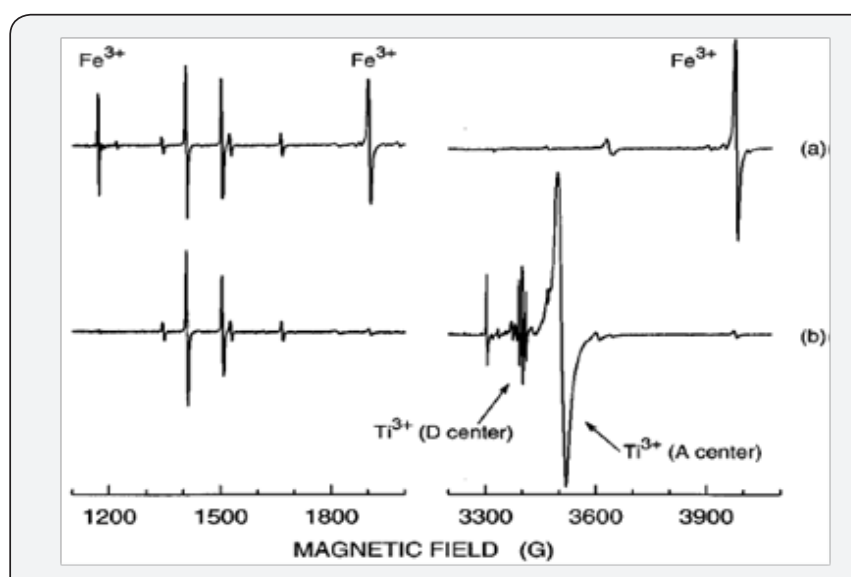

Figure 2: EPR spectra of flux-grown KTP taken at $77 \mathrm{~K}$ with the magnetic field parallel to the a axis of the crystal. The spectrometer settings were the same for each trace.

(a) Before irradiation,

(b)Immediately after an irradiation at $0^{\circ} \mathrm{C}[8]$.

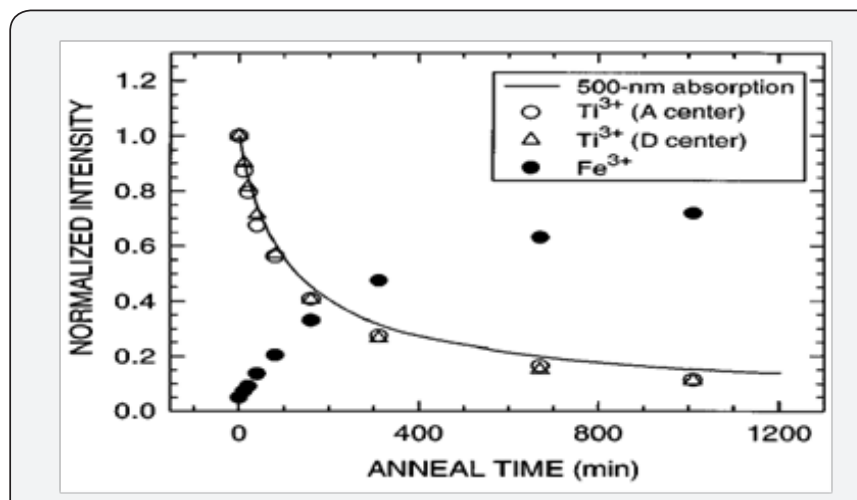

Figure 3: Recovery of flux-grown KTP.

The gray track induction produced by x-ray source was used for investigation. The gray track didn't last for more hours, they experienced recovery within $10 \mathrm{hrs}$ to the partial stage pre irradiated condition at room temperature, continuous monitoring of optical absorption at $500 \mathrm{~nm}$ wavelength for $20 \mathrm{hrs}$ showed declination, A significant decay of the EPR spectra from the two $\mathrm{Ti}^{3+}$ centres and growth of the isolated $\mathrm{Fe}^{3+}$ centres is seen in (Figure 3) [8]. All these lead to the formulation concept. The optical absorption at $500 \mathrm{~nm}$ and the EPR signals from the $\mathrm{Ti}^{3+}$ and $\mathrm{Fe}^{3+}$ centres were monitored over a period of $20 \mathrm{~h}$ at 
room temperature [8]. The chemical formulation representation given with respect to the above hypothesis on redistribution of charges is equation (1)

$$
\begin{gathered}
\mathrm{Fe}^{3+}+\mathrm{h}^{+} \stackrel{\stackrel{\text { formation }}{\longleftrightarrow}}{\stackrel{\text { decay }}{\longrightarrow}} \mathrm{Fe}^{4+}-(1) \\
\mathrm{Ti}^{4+}-\mathrm{Vo}+\mathrm{e}^{-} \stackrel{\text { formation }}{\stackrel{\text { decay }}{\longrightarrow}} \mathrm{Ti}^{3+}-\mathrm{Vo}-(2)
\end{gathered}
$$

There is an excellent correlation, as shown in (Figure 3), between the decay of the optical absorption and the decay of the two $\mathrm{Ti}^{3+}$ centres. Also, the growth of the EPR spectrum of the isolated $\mathrm{Fe}^{3+}$ centres coincides directly with the other decays. From these results, it is concluded that gray tracks are formed in KTP when isolated $\mathrm{Fe}^{3+}$ ions trap holes and $\mathrm{Ti}^{4+}$ ions (with an adjacent oxygen vacancy) trap electrons. But he nowhere addressed about the role of $\mathrm{K}^{+}$(potassium) centres which is one of the major constituent in KTP crystal though he given chemical formation and decay formula he didn't mention on the thermal effect and activation energy which is an important parameter while considering the combination and recombination but $\mathrm{V}$. Mürk [12] model of two subsystem overcame previous statement on $\mathrm{K}^{+}$ions and also given some acceptable reason for formation of these defects. From his experiments he conveyed the two subsystem based on natural KTP crystallographic structure one is the radiation-resistant oxide framework of $\mathrm{TiOPO}_{4}$, and the relatively mobile of the $\mathrm{K}^{+}$captions located in the framework cavities as the energy needed for creation of a defect in the potassium sub lattice is very less say not greater than $1.4 \mathrm{eV}$, the local lattice vibration occurs when there is overheating which is much enough for non-radioactive $\mathrm{TiO}_{6}$ octahedral exactions develop a Freckle-defect pair, $\mathrm{K}_{\mathrm{i}}^{+}-\mathrm{V}\left(\mathrm{K}^{+}\right)$, the micro volume containing $\mathrm{K}_{\mathrm{i}}^{+}$- ion capture electron and form $\mathrm{Ti}^{3+}$ colour centres and holes outside this volume create various oxygen colour centres and thereby gray tracking emerges he also experiments on bleaching process which is of great use from the time of growth of crystal will be seen in detail in session of recovery from gray tracking later.

\section{Laser Induced Gray Track}

Laser is one of the prime application of KTP but this also remain a cause for gray tracking to make it simple the purpose of it's make itself a inducer of its damage. In accordance with consideration of gray tracking as a multiphase damage there is said to be involvement of simultaneous exposure of multiple waves like, IR and visible rays. People considered gray tracking and its source cause as a mystery until mechanism of photo chromic damage as been explored, because they experienced gray track for variety of rays and situation for instance they observed gray track formation due to second harmonic generation $[2,4,13,14]$ which is neither IR nor Visible Rays, even Blachman $\mathrm{R}$ proved it with UV radiation. Studying the effect caused by these sources may lead to some revelation of formation of gray track by laser i.e. in application.
Gray tracking induced by a laser is classically studied on the basis of several types of experiments:

1. Visual observation of the darkening.

2. Observation of second-harmonic beam distortion during 1064-nm SHG [3,13].

3. Optical transmission coefficient measurement by the beam which creates the damage [5] or by a probe beam during laser exposure $[3,13,15]$.

4. Optical transmission or absorption spectra $[2,13,16$ 18],

5. Electron spin resonance (ESR) spectra [14,16] measured before and after laser irradiation.

6. From these experimental studies, some properties of the laser-induced gray-tracking have been determined $[3,13]$ :

(a) The gray tracking threshold (expressed as the laser peak intensity above which the damage is observed) is a decreasing exponential function of the Q-switch frequency [13] knowing the threshold will render a productive usage, limitation and maintenance of devices.

(b) This also help in knowing the concept that a laser beam with a polarization parallel to the polar axis of KTP, i.e., the binary axis creates more damage than a beam polarized orthogonally to the axis [2], [14] this information gives us a knowledge on positioning of Crystal.

(c) The time constant is dependent on the intensity of the laser beam as far the level of gray-tracking reaches the asymptotic value $[14,15,17,18]$. This gives the assumption about the intensity of laser and safe duration of its exposure to the crystal, thereby indicate the lifetime of crystal.

So far the revelation of these properties will implicate their careful usage and limitation of device part but elucidating the mechanism of gray track will actually help in précising parameters from growth stage itself making it superefficient and increasing its lifetime in application. Laser beam radiation in KTP produces photo carriers in the volume of the beam. Then, the electrons and holes will drift apart and become trapped separately by the defects in the crystal by following the possible mechanism as mentioned before. The actual application of this crystal is to transmit secondary harmonic energy waves, today third harmonics is also being in existence effectively for use from the incidental fundamental waves, these waves are with adequate peak power intensity for respective applications this beyond certain limit (threshold) seem to cause the gray tracking, detail studies of these is as follows:

\section{Dependencies of Gray Tracking}

For any damage or growth process there are factors which influence them, these should be taken into consideration for 
successful product and for reduction of blunder, the various condition and dependent factors influencing the gray track is as follows.

\section{Wavelength and Threshold}

Experiments of Boulanger B [19] on KTP regarding the wavelength and intensity peak power and proved the dependency of gray tracking, He tried out with 1064nm, 532nm, $355 \mathrm{~nm}$ where many focussing only on SHG of $532 \mathrm{~nm}$ his team worked hardly on proving the influence of $355 \mathrm{~nm}$ which they considered as a possible THG, went in vain because ultimately they ended up with result that neither $1064 \mathrm{~nm}$ nor $355 \mathrm{~nm}$ have no influence on inducing gray tracking in contrast to report of Blachman R [20] and in accordance with report of Loiacono GM [2] but their investigation on intensity peak power gave an important parameter for consideration i.e. the threshold value of intensity peak power $80 \mathrm{MW} / \mathrm{cm}^{2}$ above which darkening of crystal start appearing. As a known fact electron excitation from the valence band to the conduction band action is responsible for any kind of reaction occurring say here as harmonic generation, there is need of energy for excitation and transmission Thus there is requirement of constant high power of laser that is also one of the inducing factor of damage that cause astigmatism of SHG efficiency and finally result in catastrophic damage so in below two subtopics dependencies of alternating solutions where discussed.

\section{Repetition Rate of Laser}

The alternative solution for the blame of constant high power that reduce the lifetime of crystal rapidly is providing them in pulse mode, that leads to another considerable factor repetition rate, It seem to decrease the threshold of gray tracking, previously the threshold value was mentioned as $80 \mathrm{MW} / \mathrm{cm}^{2}$ which was under weak focussing whereas to the experimental evidence of Fève JP [21] that the gray-tracking peak power threshold strongly decreases when the repetition rate is increased: from $125 \mathrm{MW} / \mathrm{cm}^{2}$ at $1 \mathrm{kHz}$ to $18 \mathrm{MW} / \mathrm{cm}^{2}$ at $6.3 \mathrm{kHz}$, and to a few $\mathrm{MW} / \mathrm{cm}^{2}$ for frequencies greater than $10 \mathrm{kHz}$. Therefore the threshold itself depends on frequency and focussing condition. These strong focussed crystal take lot time say more than a year to recover from the gray tracking. Qiuhui Zhang [22] also conducted studies on high repetition rate of laser in KTP and devised some useful model. As mentioned before once gray tracking started it continuously accumulates and ends at catastrophic damage. He devised a model on accumulation of gray tracking once it is started i.e. once colour centres formation initiated with according to the mechanism previously discussed, the bond breaks incessantly and absorption increases, the density of colour centres contribute to gray tracking centres, So there comes the relationship with variation in colour centres which is given by the number of colour centres $\Delta n[23,24]$.

$$
\Delta n=D F_{o}^{m} k_{B} T \exp \left[-\frac{\left(U_{0}-\gamma \sigma\right)}{k_{B} T}\right]
$$

where D- is a constant of proportionality, F0 -the peak photon flux density of the incident, $\mathrm{m}$ - the order of multi-photon absorption, $\mathrm{k}_{\mathrm{B}}$-the Boltzmann constant, $\mathrm{T}$ - the temperature, U0 -the initial activation energy of damage which is found to be $1-3 \mathrm{eV}$ for various materials, $\gamma$ the material parameters, and $\sigma=\sigma_{T}+\sigma_{c}$ the stress which includes thermal stress $\sigma_{T}=C \alpha K F_{0}^{m}$ (C: a constant, $\alpha$ : the thermal expand coefficient, K: the bulk modulus of crystal) and the stress $\sigma_{c}=K n_{c} v\left(n_{c}\right.$ : the density of colour centres, $v$ : the volume of each ion) induced by lattice expand owing to colour centres. This colour centres accounts primarily to gray tracking not the catastrophic damage, including gray tracking other factors (for e.g., thermal stress) that has been excluded from the equation may also add to the catastrophic damage. Including the above term with the nF- initial colour density (The density of absorption centre can also be estimated by Macula formula [25]), V- the volume of the focus region, is the change of colour centres after an infinite time, $r$ is a constant describing the rate of relaxation, $t$ is the time, It gives the density of gray tracking after each pulse as below

$$
\rho_{1}=\frac{n_{F} V+\Delta n-\Delta N[1-\exp (-r t)]}{V}
$$

After each irradiation there will be summation of density term with change in exponential term alone which is seen after $2^{\text {nd }}$ irradiation,

$$
\rho_{2}=\frac{n_{F} V+\Delta n-\Delta N[1-\exp (-r t)}{V}+\frac{n_{F} V+\Delta n-\Delta N\left[1-\exp \left(-r\left(t-\frac{1}{f}\right)\right]\right.}{V}
$$

Similarly $\mathrm{n}^{\text {th }}$ irradiation is given by,

$$
\rho_{n}=\frac{n_{F} V+\Delta n-\Delta N[1-\exp (-r t)]}{V}+\frac{n_{F} V+\Delta n-\exp \left(-r\left(t-\frac{(n-1)}{f}\right)\right]}{V}-(6)
$$

$\mathrm{f}$ is the repetitive rate of incident pulses, $\mathrm{t}$ the lasting time of irradiation. This iteration ends up i.e. the saturation of gray tracking occurs only if $U_{0} \geq \gamma \sigma$ i.e. the stress induced by the expansion of colour centres is lesser than atom binding energy, this can be made as indication for the extent the crystal withstand before the entire damage, Using this one can simulate the gray tracking formation in crystal.

\section{Influence of Impurities}

Next alternative is adding impurities sometimes intentionally in name of do pant to enhance the property of material for instance: Ce improves the KTP transparency, trivalent metal cation are used to decrease the electrical conductivity along the $c$ axis, $\mathrm{Nb}^{5+}$ shifts the second harmonic generation cut-off to shorter wavelengths, and also to reduce the required power and thus improving the threshold of gray tracking. C.Zaldo $[26,27]$ one among major contributors involved in the do pant investigation, explored by incorporating different do pants like $\mathrm{Zr}, \mathrm{Er}, \mathrm{Na}, \mathrm{W}, \mathrm{Ho}, \mathrm{Nd}, \mathrm{Nr}$, and dual composition (Wr:Rh) such different dopants of particular interest was tested UV, and x-ray irradiation but if doped samples does not meet the requirement say like inducing it by photons with energy lower than the KTP 
band gap [28] they also suffer from damage. He and his team experimented on both doped and undoes KTP and thermally bleached (It is a thermal treatment which involves heating the sample above the room temperature to reproduce the crystal to the state before irradiation) heating the crystal doped samples. They presented following information for us:

A. The presence of impurities will induce two changes:

B. Appearance of a band at about $488 \mathrm{~nm}$ especially for high concentration of impurity

C. Shift of Optical absorption edges which solely depend on concentration of impurities, they viewed that the shift is caused due to local lattice distribution and stress created by impurities.

D. The growth sectors of 001 of KTP incorporate more impurities comparatively because this axis has structural channels which eases the ion exchange and impurity diffusion

E. Damage induced in doped samples has higher thermal stability in comparison with undoped sample.

F. They also tried the trapping efficiency of electron though they made a statement that it is very difficult because it differ for each impurity.

G. They also devised a model for kinetic damage and erasure. With this as reference one can gain information and predict changes that could happen on addition of impurities, hence there would be some conceptual knowledge in prior and reduce the redundancies [29].

\section{Depth}

The radiation damage depend on depth of impurities is tested with $\mathrm{Li}^{+}, \mathrm{N}^{+}, \mathrm{Ar}^{+}, \mathrm{Er}^{+}, \mathrm{B}^{+}$and many, where each ion have their own effect means here the depth of affection layer with respect to the energy loss in nuclear or electron range in the complex structure of crystal consisting of covering layer, transition layer of amorphous cluster and buried amorphous layer respectively, Where comes the role of energy there comes the play of threshold, this discussion is also not a exempt to it, threshold value of the electronic energy deposition(natural energy that an ion posses in electron level) from the analysis of data seems to be $100 \mathrm{eV} /$ ion/ Å. Each ion depend on their energy effectively take their place among the layered structure, Among others B+ seems to dwell deeper into the structure and $\mathrm{Li}+$ was on superficial layer which again emphasis on the energy of ion [30].

\section{Polarisation}

The study related to dependence of gray-track susceptibility with respect to polarization was conducted by Hua XB [6], they irradiated light in directions perpendicular and parallel to the polar axis of KTP crystals. He explains the lifetime of susceptibility incoherence with mechanism responsible for formation of gray tracking, the longer the lifetime of electrons and holes, the higher will be the crystals susceptibility to gray tracking. Though the source here is laser, involvement of electron and hole significantly affected by the electric field, as the topics were separated based on source you encounter this here. There are structural channels parallel to the polar z-axis through which the $\mathrm{K}+$ ion can easily move under the influence of an electric field by a hopping mechanism, leading to a high ionic conductivity along this direction [31,32]. They focused only on role of holes to the orientation in parallel and perpendicular direction, when a light beam is propagated in a crystal, the optical frequency electric field would interact with the charges in the crystal.

If the optical frequency electric field is parallel to the z-axis, the $\mathrm{K}$ ions will hop along the structural channel due to the high ionic electric conductivity in this direction. In this case, $\mathrm{K}$ vacancies do not localize at a definite position and will not serve as a stabilizing entity for the whole trap. In other words, the whole trap will become destabilized and have a short lifetime. As a result, the optical absorption centres will annihilate as soon as they are produced when the optical frequency electric field is parallel to the z-axis. In contrast, when the optical frequency electric field is perpendicular to the z-axis, the hole trap will be stabilized because the potassium vacancy can keep its position and provide a stabilizing force for the hole trap, i.e. the hole trap will have a long lifetime in this case [13]. To conclude the laser irradiation in the direction for which optical frequency, electric field will be in ||z polarization give a rare chance to gray tracking irrespective of exposure time.

\section{Control of Gray Tracking Formation in KTP Crystal}

Gray tracking formation before laser irradiation depend on growth technique and factors like environment, temperature, flux and impurities, To the fact 'As stitch in time saves nine' controlling at early stage in easy and deduce post work and also provide efficient result. Research by Perlov D [33] investigated temporal and spatial dimension of gray tracking for quantitative evaluation of KTP gray track susceptibility with respect to the factor, they reported the first experimental evidence of dependency on the material Curie temperature and electrical conductivity, and they observed the susceptibility of KTP could be significantly decreased by adjusting the growth parameters. For instance impurities included in crystal would originate the optical absorption where in case susceptibility also depend on absorption coefficient before laser irradiation this event add to the cause so that one can understand that crystal with many impurities indicated large susceptibility but exceptionally crystal with high concentration of hydroxyls had low susceptibility. This lead to the understanding the role of moisture in preventing gray tracking.

Considering this concept Shinji Motokoshi [34,35] tried to improve the gray-tracking by controlling the hydroxyl concentration in KTP crystal using the thermal annealing process. It was found that large GTS (gray tracking susceptibility) in original crystal was improved by annealing in the air and that was almost returned to original value by annealing in vacuum. These results mean that the change of GTS was caused not only 
by an effect of the heating, but also by the transfer of molecules in the air such as oxygen and hydroxyl. However, it was previously reported that the annealing did not influence to the gray-tracking and the hydroxyl. The reason of difference with their data was due to the annealing conditions. The optimization of annealing conditions such as temperature, time, and environment had been investigated and a new approach proposed was thermal annealing pre-process. It was demonstrated that the graytracking formation and the hydroxyl concentration could be controlled by thermal annealing process before laser irradiation, the KTP crystal was decreased transmittance with the laser irradiation time before the annealing.

In the case of the annealing in air, however, the decrease of transmittance was suppressed, and also the transmittance decreased again by annealing in the vacuum as mentioned before, As a result they understand that KTP crystal could include the hydroxyls in air by the annealing process. In this work, it was shown the dependence of gray-tracking susceptibility on preannealing conditions as atmosphere gases, temperature and humidity for KTP. The annealing was made with an electric oven at the temperature up to 1000 0C. The atmosphere in the oven was exchanged to air, nitrogen, oxygen, and vacuum (at 10-3 torr). The humidity in nitrogen gas was changed from 6 to $99 \%$ by control the flow rate both dry and wet gases. The annealing time in vacuum was 72 hours, and the others were 12 hours $[34,35]$. In comparison with no annealed sample, the samples annealed in air and N2-wet indicated decreased gray-tracking formation. The contrast occurred while annealing them in vacuum and dry gases. This cleared that gray-tracking formation could be controlled by humidity in gases but not the kind of gases. Thus one can understand the dependency and positive application of impurity well and thus efficient control is made easy.

\section{Elimination of Gray-Tracking Effects of $\mathrm{KTiOPO}_{4}$}

Controlling make the KTP resistant to gray track to increase the life time of it in application, where elimination is the process done after the appearance of gray track to the extent until it meet the catastrophic damage for the purpose of reuse, Generally two methods are followed to eliminate the chromic damage which is explained in following sessions [36].

\section{Thermal Annealing}

Thermal annealing is the general and simple, effective processes to retrieve the original state of crystal by eliminating the gray tracking, The temperature depend on density of gray tracking formed so the temperature differs in each crystal and approximating it prior is difficult, from our analysis of multiple data mostly KTP recovered from gray tracking was annealed about temperature above $150{ }^{\circ} \mathrm{C}$.

\section{Strong Focussing Mechanism}

There was discussion on some dependencies previously there one could find the dependency and relation of radiation beam radius, focussing and power density in formation of gray tracking and among them respectively. Thus it is known that radius of beam and power density are inversely proportional, XIANG Zhen [30] proposed a system based on the above concept that eliminate the formed gray track. They thus made a setup as in below figure (Figures 4-6) where the radius of input beam is enlarged this technique resulted in decrease of power density so as decrease in conversion efficiency. Therefore it resulted in condition that one should compromise between conversion efficiency and deformation of gray tracking, when they went into detail analysis of any alternate way they found one thing in common that the increase in radius within the KTP only provided the required changes. So they adjusted the setup by adjusting the lens L2 of the focussing system thus the radius of incident beam on surface is small but in enlarges inside the crystal this provided the satisfactory result with un changed conversion efficiency and this idea of enlarging the beam only at output surface is strong focussing and both output and input is weak focussing. However effective may the strong focusing the large divergence in crystal may lead to phase mismatching, Luckily KTP has large acceptance angle that prevent this but careful choosing on focus ratio and the distance between the crystal and focussing system to be made to avoid discrepancies, else one may phase consequence like decrease in efficiency due to phase matching and appearance of gray track.

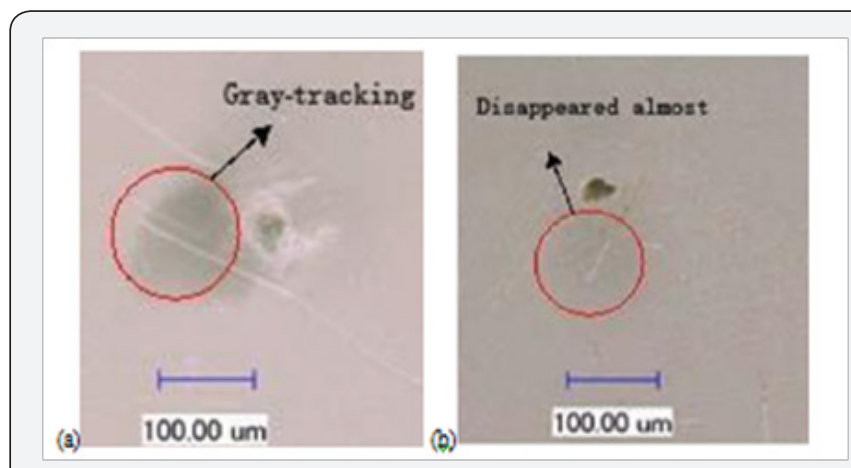

Figure 4: (a) Appearance of gray tracking,

(b) Disappearance of gray tracking [15].

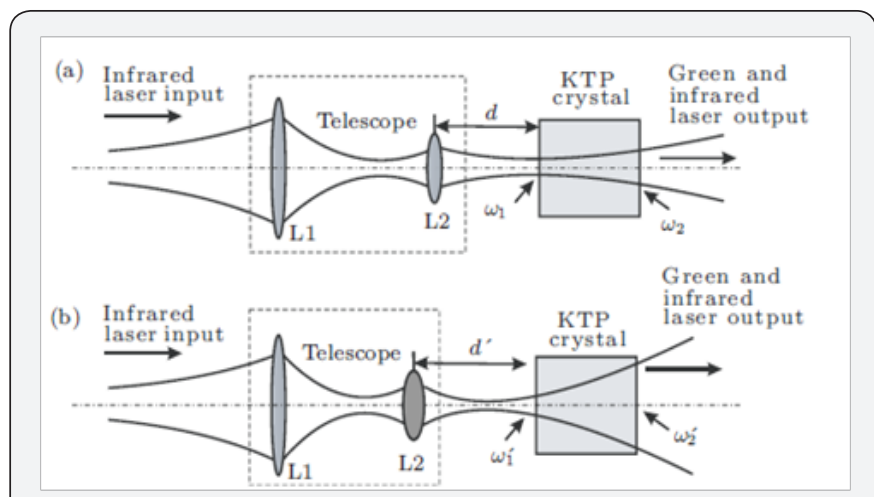

Figure 5: Setup of (a) weak focussing,

(b) Strong focussing [30].

\section{Electrically Induced Gray Track}

Any material finally reaches the application status which involves electrical device and application of electric field, the 
application of an electric field leads to damage by electrolysis i.e. by electrical currents flowing through the crystal. For instance: For dc fields; $0.4 \mathrm{kV} / \mathrm{cm}$ along the polar axis, a grey colouration is observed and this is known as electric field induced gray tracks [31]. Furthermore, at ac fields of; $0.4 \mathrm{kV} / \mathrm{cm}$ and $1 \mathrm{~Hz}$ frequency, there is induced order-disorder phenomenon involving $\mathrm{K}+$ ions have been observed. The schematic diagram of experimental set up electrically inducing of gray track shown [40]. The crystal structure of KTP (space group Pna21) consists of threedimensional network of $\mathrm{TiO} 6$ octahedral and $\mathrm{PO}^{4}$ tetrahedral [32]. There are structural channels parallel to the polar c axis through which the $\mathrm{K}^{+}$ions can easily move under the influence of an electric field by a vacancy mechanism, (similar to one of two sub system modelled by Murk V [7] leading to a high ionic conductivity along this direction emphasized by Morris PA [33]. Gray tracks produced in $\mathrm{KTiOPO}^{4}$ (KTP) by applying a dc electric field have been studied through optical absorption, Raman scattering, and synchrotron x-ray topography. The study of the optical absorption and Raman scattering from the gray-tracked region suggests that their formation is accompanied by changes in the electronic levels of $\mathrm{Ti}^{+}$and also provided information on remnants strain of gray tracking [34-36].

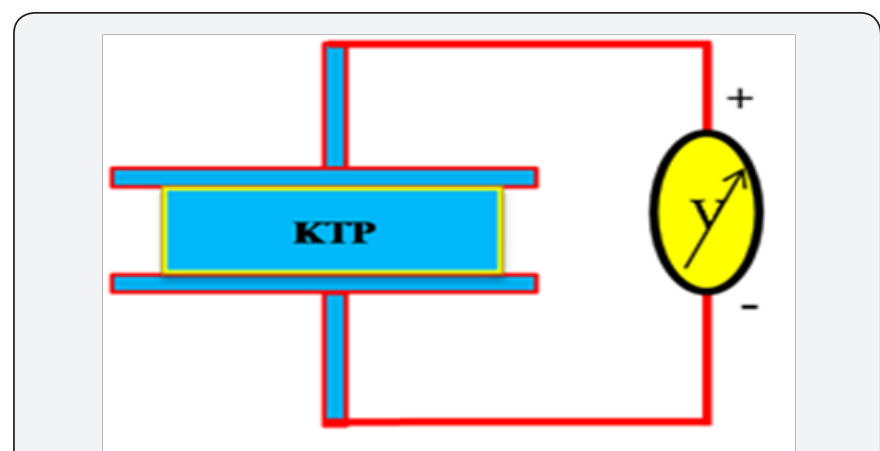

Figure 6: The schematic diagram of experimental set up electrically inducing of gray track [40]

\section{Remnant Strain of Gray Tracking}

Raman spectra of KTP crystals containing gray tracks were obtained by passing the laser beam through the gray-track region. The x-ray topography of the gray-track region was recorded using the synchrotron radiation source operating at $2 \mathrm{GeV}$ with $250 \mathrm{~mA}$ current. Satyanarayan MN [37] aim was to identify the localized structural changes or disorder that may occur along the grey tracks. In their observation they found gray colouration which obviously due to absorption but in region of $400-850 \mathrm{~nm}$ to achieve their aim they should check the effects of absorption and Raman spectrum, all there reports were in comparison with the virgin and gray tracked crystal, so the resulted Raman intensity was reduced for gray tracked crystal than the virgin which is due absorption but the normalised intensity was greater for gray tracked crystal, so looked for other parameter influencing the Raman intensity. Such as that wavelength of incident radiation and the electronic energy level structure in the crystal seems to play a role, He found that for lower wavelength still lower comparative values were obtained than the previous which impose a direct relationship. If there is any structural change or disorder in the crystalline state then it gives rise to the new Raman lines and frequency shifts.

The existing Raman lines might also split, if there is a localized disorder and translational symmetry is destroyed within the crystal. Here in their experimental research they didn't find any such features, so they strongly believe and recommend that no major structural changes is happening to the crystal due to electrically induced gray track but this is not the only criteria to confirm it, further investigation with inclusion several condition is necessary to come to an conclusion. They also proposed a idea on comparing the structural damage due to both ac and dc field, where the work is not yet published, However from the so far investigation there is only revelation of electronic level changes which is relating to dc field. Nevertheless, the x-ray topography indicates that a remnant strain persists along the gray tracks [37].

\section{Influence of water vapour in electrical damage}

The moisture influence was seen in recovering the gray tracking in crystal before applying it as a device, Here its influence in electro-optic device. The research work of Morris PA and Crawford MK investigation results indicate that the susceptibility of hydrothermal and flux grown KTP crystals to electric-field damage is increased when the atmosphere surrounding a device has a relatively high vapour pressure of water. Protons from the atmosphere migrate into the crystals due to the applied electric field and provide charge compensation for the Ti3+ defects responsible for damage in KTP [38]. Here they compare two growth technique one is hydrothermal and another is flux growth, among them flux grown is highly susceptible to the electric field damage. This is happening due to migration of Hydrogen from atmosphere to the crystal via cathode, some researchers also observed yellow colour induced at anode due to excess electron [38]. Morris PA [39] made significant contribution in investigating electro chromic damage of KTP by analysing many parameters like temperature, optical properties and also the possible potassium vacancy system at very early stage, among them most minute and significant is the relation of AC conductivity with the gray tracking [40-44]. The creation of damage is directly proportional to the average current which in turn proportional to the AC conductivity of crystal, the crystal's conductivity might increases due to impurity addition. This damage leads off at cathode and migrates to anode until the entire crystal gets damaged.

\section{Conclusion}

This article covers broad scope of gray-tracking, this even helps amateur to acquire knowledge on it because this explains the phenomenon of gray-tracking including its effects in product ,causes for formation, possible mechanism in crystal that employ gray-tracking such as laser induced,electrically induced and defects due to its growth technique, polarisation effects and 
influence of impurities and its depth and this mostly concentrate on KTP crystal which is a promising superior material for a variety of nonlinear optical and electro-optical applications. It also direct your way of search if interested in further investigation on gray tracking.

\section{References}

1. Beno^ıt Boulanger, Jean-Philippe Feve, and Yannick Guillien (2000) Thermo-optical effect and saturation of nonlinear absorption induced by gray tracking in a 532-nm-pumped KTP optical parametric oscillator . Optics Letters 25(7): 484-486.

2. Loiacono GM, Loiacono DN, Mc Gee T, Babb M (1992) J Appl Phys 72: 2705.

3. Jacco JC, Rockafellow DR, Teppo EA (1991) Bulk-darkening threshold of flux-grown $\mathrm{KTiOPO}_{4}$. Opt Lett 16(17): 1307-1309.

4. Roelofs MG (1989) Identification of $\mathrm{Ti}^{3+}$ in potassium titanyl phosphate and its possible role in laser damage. J Appl Phys 65(12): 4976.

5. Andreev BV, Maslov VA, Mikhailov VA, Pak SK, Shaunin OP, et al. (1991) SPIE Solid State Lasers and New Laser Materials 1839: 280-289.

6. Hua XB, Wang JY, Zhang HJ, Jiang HD, Liu H, et al. (2003) Journal of Crystal Growth 247: 137-140.

7. Scripsick MP, Edwards GJ, Halliburton LE, Belt RF, Loiacono GM (1994) J Appl Phys76: 773.

8. Scripsick MP, LoIacono DN, Rottenberg J, Goellner SH, Halliburton LE, et al. (1995) Appl Phys Lett 66(25): 3428.

9. Nizamutdinov NM, Khasanova NM, Bulka GR, Vinokurov VM, Rez IS, et al. (1987) Sov Phys Crystallogr 32: 408.

10. Stenger JF, Dusausoy Y, Marnier G, Rager H, Gaite (1989) Electron paramagnetic resonance study of a new $\mathrm{Fe}^{+}$centre in $\mathrm{KiTiOPO}_{4}$. JM J Phys Condens Matter 1(28): 4643.

11. Gaite JM, Stenger JF, Dusausoy Y, Marnier G, Rager H (1991) Electron paramagnetic resonance study of paramagnetic defect centres $\mathrm{Fe}^{3+}$ and $\mathrm{Cr}^{3+}$ in $\mathrm{KTiOPO}_{4^{4}}$ J Phys Condens Matter 3(40): 7877.

12. Mürk V, Denks V, Dudelzak A, Proulx PP, Vassilitsenko V (1998) Nuclear Instrum Methods Phys Res 141: 472.

13. Blachman R, Bordui PF, Fejer MM (1994) Appl Phys Lett 64: 1318-1320.

14. Andreev BV, Maslov VA, Mikhailov VA, Pak SK, Shaunin OP, et al. (1991) SPIE Solid State Lasers and New Laser Materials 1839: 280-289

15. Voitsekhovii VN, Yakobson VE, Kalintsev AG, Kaminskii SV (1995) J Opt Technol 62: 777-779.

16. Martin MJ, Zaldo C, Diaz F, Sole R, Bravo D (1995) Radiat Eff Defects Solids 136(1-4): 243-247.

17. D'yakonov GI, Maslov VA, Mikhailov VA, Pak SK, Shaunin OP, et al. (1990) Meeting Advanced Solid-State Lasers Utah 213-218.

18. Angert NB, Garmash VM, Pavlova NI, Tarasov AV (1991) Sov J Quantum Electron 21: 426-428.

19. Boulanger B, Rousseau I, Féve JP, Maglione M, Ménaert B, et al. (1999) IEEE Journal Of Quantum Electronics 35(3).
20. Blachman R, Bordui PF, Fejer MM (1994) Appl Phys Lett 64: 1318-1320.

21. Feve JP, Boulanger B, Marnier G, Albrecht H (1997) Appl Phys Let 70: 1-3.

22. Qiuhui Zhang, Guoying Feng, Jinghua Han, Binhou Li, Qihua Zhu et al. (2011) Optik 122 1313- 1318.

23. Casper RT, Jones SC, Braunlich P, Kelly P (1990) Nucl Instrum Methods. Phys Res Sec 46: 231-234.

24. Jones SC, Braunlich P, Casper RT, Shen XA, Kelly P (1989) Opt Eng 28: 1039-1068.

25. Shu G Fang, Zhang S, Qi R (1989) The Physics of Crystal Color Center.

26. Zaldo C, Carvajal J, Solé R, Dỉaz F, Bravo D, Kling A (2000) J Appl Phys $88(6)$.

27. Solntsev VP, Tsvetkov EG (1997) Inorg Mater Transl of Neorg Mater 33:1055.

28. Martı́n MJ, Zaldo C, Dı́az F, Sole' R, Bravo D, et al. (1995) Radiat Eff Defects Solids 136: 243.

29. Maslov VA, Mikhailov VA, Shaunin OP, Shcherbakov IA (1997) Quantum Electron 27: 356.

30. Schrempel F, Höche Th, Ruske JP, Grusemann U, Wesch W (2002) Nuclear Instruments and Methods in Physics Research B 191: 202-207.

31. Hamichi M, Cousins CSG, Meads RE, Sheldon BJ, Teat SJ, et al. (1994) J Appl Phys 76: 188.

32. Furusawa S, Hayasi H, Ishibashi Y, Miramoto A, Sasaki T (1993) J Phys Soc Jpn 62: 183.

33. Perlov D, Loiacono D, Czechowicz P Crystal associates member of coherent photonics group NJ07936.

34. Motokoshi S, Jitsuno T, Izaw Y, Nakatsuka M CMU 7.

35. Motokoshi S, Jitsuno T, Izaw Y, Nakatsuka M (2000) proc of boulder damagea symp. XXXI, SPIE 3902(2000)423: proc of ahpla, SPIE 3889(2000): 644 .

36. Xiang Zhen, Jian Hong GE, Zhao Zhi Gang, Wang Sha, Miao HU, et al. (2009) Chin Phys Lett 26(8): 084202.

37. Satyanarayan MN, Bhat HL, Srinivasan MR, Ayyub P, Multani MS (1995) Appl Phys Lett 67(19).

38. Morris PA, Crawford MK (1993) Appl Phys Lett 62.

39. Morris PA, Crawford MK, Ferretti A, French RH, Roelofs MG, et al. (1989) Mat Res Soc Symp Proc 152.

40. Sadhasivam S, Rajesh Narayana Perumal, Ramasamy P (2015) Journal of Crystal Growth 431: 32-38.

41. Bosenberg WR, Guyer DR (1992) Single-frequency optical parametric oscillator. Appl Phys Lett 61(4): 387.

42. Bordui PF, Fejer MM (1993) Ann Rev Mater Sci 23: 321.

43. Tyminski JK (1991) J Appl Phys 70: 5570.

44. Dudelzak A, Proulx PP, Denks V, Mürk V, Nagirnyi V (1999) Anisotropic fundamental absorption edge of $\mathrm{KTiOPO}_{4}$ crystals. Journal of Applied Physics 87(5). 
(C) Commons Attribution 4.0 Licens (C) DOI: 10.19080/JOJMS.2017.03.555612
Your next submission with Juniper Publishers will reach you the below assets

- Quality Editorial service

- Swift Peer Review

- Reprints availability

- E-prints Service

- Manuscript Podcast for convenient understanding

- Global attainment for your research

- Manuscript accessibility in different formats ( Pdf, E-pub, Full Text, Audio)

- Unceasing customer service

Track the below URL for one-step submission https://juniperpublishers.com/online-submission.php 